\title{
Production of Hyaluronic Acid from Molasses by Streptococcus thermophilus TISTR 458
}

\author{
Aurasorn Saraphanchotiwitthaya ${ }^{1,2, *}$ and Pattana Sripalakit ${ }^{2,3}$ \\ ${ }^{I}$ Department of Pharmaceutical Technology, Faculty of Pharmaceutical Sciences, Naresuan University, \\ Phitsanulok 65000, Thailand \\ ${ }^{2}$ Pharmaceutical Biotechnology Research Unit, Faculty of Pharmaceutical Sciences, \\ Naresuan University, Phitsanulok 65000, Thailand \\ ${ }^{3}$ Department of Pharmaceutical Chemistry and Pharmacognosy, Faculty of Pharmaceutical Sciences, \\ Naresuan University, Phitsanulok 65000, Thailand
}

('Corresponding author's e-mail: aurasorns@nu.ac.th)

Received: 21 September 2020, Revised: 6 May 2021, Accepted: 21 May 2021

\begin{abstract}
The purpose of this study was to investigate the capability of Streptococcus thermophilus TISTR 458 on hyaluronic acid (HA) production by using various carbon sources including glucose, sucrose and sugarcane molasses. HA production was determined using a turbidity assay by precipitation with cetyltrimethylammonium bromide (CTAB) and the sugar content was analysed by a sulphuric acid-UV spectrophotometry method. The results showed that $S$. thermophilus TISTR 458 produced the maximal yield of HA from glucose, sucrose and molasses at approximately $32.80 \pm 4.27(24 \mathrm{~h}), 79.03 \pm 8.36(24 \mathrm{~h})$ and $213.44 \pm 76.79(12 \mathrm{~h}) \mathrm{mg} / \mathrm{L}$, respectively, then decreased gradually. Moreover, an elevated level of HA was related to a reduction in the sugar content. It was concluded that $S$. thermophilus TISTR 458 can produce HA by using glucose, sucrose and molasses. Interestingly, molasses as a cheap agricultural byproduct is a promising prospect carbon source for HA production by $S$. thermophilus TISTR 458.
\end{abstract}

Keywords: Hyaluronic acid, Streptococcus thermophilus TISTR 458, Glucose, Sugarcane molasses, Fermentation

\section{Introduction}

HA, a linear anionic mucopolysaccharide occurring naturally throughout the body, consisted of a repeating disaccharide unit of D-glucuronic acid and $N$-acetylglucosamine linked alternately by $\beta(1 \rightarrow 3)$ and $\beta(1 \rightarrow 4)$ glycosidic bonds [1,2]. Due to its viscoelasticity, high biocompatibility, low immunogenicity and toxicity [1,3], HA has been widely used in pharmaceutical applications such as synovial fluid supplement, ophthalmological surgery, wound healing and drug delivery $[1,4]$. Moreover, it has been used in cosmetics as moisturizing agents, hydrating agents and antiaging materials [3]. HA has been conventionally produced from the extraction of rooster combs and bovine vitreous humour. However, there are some limitations of the isolation process at an industrial scale and the argument in ethical issues [2]. Therefore, various studies on HA production using microbial fermentation such as $S$. zooepidemicus [5-7] and S. thermophilus [8,9] have been reported.

Streptococci are fastidious lactic acid bacteria [10] which demands nutritional enrichment in culture medium for cell growth and HA production [11]. Using of the chemically defined medium (CDM) [10] and the addition of amino acids such as arginine and lysine in the culture medium help supportive for cell growth and HA production [12]. These lead to high production cost which was the obstacle of commercial competitiveness on HA production. To decrease the production cost, employing low-cost carbon, nitrogen sources and essential supplements such as agro-industrial byproducts in the cultivation medium was one of the significant targets for HA production at the industrial level. This study aimed to evaluate the production of HA by $S$. thermophilus TISTR 458, the culture collection from Thailand, using sugarcane molasses as low-cost carbon sources compared with glucose and sucrose. 


\section{Materials and methods}

Strains and chemicals

S. thermophilus TISTR 458 was purchased from the Thailand Institute of Scientific and Technological Research (Pathum Thani, Thailand). Standard HA and glucose were purchased from Sigma-Aldrich Chemical Co. (St. Louis, Mo, USA). The sugarcane molasses was obtained from Phitsanulok Sugar Factory (Phitsanulok, Thailand). MRS broth, MRS agar, sucrose and yeast extract were purchased from HiMedia Laboratories (India) and other reagents were of analytical grade and purchased from Merck (Germany).

\section{Preparation of stock cell suspensions}

Lyophilized powder of $S$. thermophilus TISTR 458 was suspended in MRS broth and transferred to MRS agar, incubated under an anaerobic condition at $45{ }^{\circ} \mathrm{C}$ for $48 \mathrm{~h}$. The inoculum was prepared by transferring 1 full loop of $S$. thermophilus TISTR 458 cultures from MRS agar into $250 \mathrm{~mL}$-Erlenmeyer flasks containing $100 \mathrm{~mL}$ of MRS broth. These flask cultures were incubated under conditions at $150 \mathrm{rpm}$ (revolutions per minute), $28 \pm 2{ }^{\circ} \mathrm{C}$ for $48 \mathrm{~h}$ on an orbital shaker (SK3PO, CT laboratory, USA).

\section{Effect of different carbon sources on hyaluronic acid production}

The basal biotransformation medium was composed of glucose 30 , yeast extract $30, \mathrm{~K}_{2} \mathrm{HPO}_{4} 2.5$, $\mathrm{NaCl} 2.0$ and $\mathrm{MgSO}_{4} \cdot 7 \mathrm{H} 2 \mathrm{O} 1.5 \mathrm{~g} / \mathrm{L}, \mathrm{pH}$ 6.8. To study the effect of carbon sources on HA production, glucose was replaced with sucrose or sugarcane molasses. Sterile biotransformation medium with an initial volume of $90 \mathrm{~mL}$ in $250 \mathrm{~mL}$-Erlenmeyer flasks was inoculated with $10 \mathrm{~mL}$ stock culture of $S$. thermophilus TISTR 458 and cultured under $37 \pm 2{ }^{\circ} \mathrm{C}$ with occasionally shaking for $72 \mathrm{~h}$. During the biotransformation process, $1 \mathrm{~mL}$ of the culture medium was collected at $0,3,6,9,12,24,48$ and $72 \mathrm{~h}$ for analysis of HA and sugar.

\section{Determination of hyaluronic acid by the turbidity assay}

Quantitative analysis of HA in the culture was determined using CTAB turbidimetric method. The collected fermented broth was centrifuged at 9,000 rpm for $15 \mathrm{~min}$. Fifty microliters of the supernatant were placed into 96 -well plate, mixed with $50 \mu \mathrm{L} 0.2 \mathrm{M}$ acetate buffer $(0.2 \mathrm{M}$ sodium acetate-acetic acid, $0.15 \mathrm{M}$ sodium chloride, $\mathrm{pH} \mathrm{6}$ ) and incubated at $37^{\circ} \mathrm{C}$ for $15 \mathrm{~min}$. After that, $100 \mu \mathrm{L}$ of $2.5 \% \mathrm{w} / \mathrm{v}$ CTAB in $2 \% \mathrm{w} / \mathrm{v}$ sodium hydroxide was added and mixed well with a vortex mixer. The mixture was incubated at $37{ }^{\circ} \mathrm{C}$ for $10 \mathrm{~min}$. The turbidity of the mixture was measured by a microplate reader at $600 \mathrm{~nm}$ within $10 \mathrm{~min}$ and the HA content was calculated [13]. The calibration curve of HA standard solution at concentrations between $0.1-0.5 \mathrm{~g} / \mathrm{L}$ showed good linearity. The regression line for HA was described by the following: $\mathrm{Y}=0.93 \mathrm{x}+0.002\left(\mathrm{R}^{2}=0.999\right)$, where $\mathrm{Y}$ is OD value and $\mathrm{X}$ is the concentration of HA $(\mathrm{g} / \mathrm{L})$.

\section{Determination of glucose by the spectrophotometric method}

Glucose was determined by the sulfuric acid-UV-Vis spectrophotometry [14], with a slight modification. Five hundred microliters of the supernatant of fermented medium or distilled water (blank) and $1.5 \mathrm{~mL}$ sulfuric acid were added in the test tube and rapidly mixed well by a vortex mixer for $30 \mathrm{~s}$ to complete the reaction. After that, each test tube was cooled down in ice for 2 min to room temperature. The OD value was measured by a microplate reader at 297 and $316 \mathrm{~nm}$ for glucose and sucrose, respectively. The calibration curve of a glucose solution at concentrations between $0.01-0.09 \mathrm{~g} / \mathrm{L}$ showed good linearity. The regression line was described by the following: Glucose $\mathrm{Y}=13.409 \mathrm{X}$ $0.1535\left(\mathrm{R}^{2}=0.9958\right)$; sucrose $\mathrm{Y}=9.8744 \mathrm{X}+0.086\left(\mathrm{R}^{2}=0.9989\right)$, where $\mathrm{Y}$ is absorbance and $\mathrm{X}$ is concentration of glucose or sucrose $(\mathrm{g} / \mathrm{L})$.

\section{Determination of cell growth}

The collected culture medium of $100 \mu \mathrm{L}$ at each determined time was diluted 10 times with distilled water. Cell growth was determined by cell enumeration using a hemocytometer under a microscope (40X). The dynamics of cell growth was represented by plotting the cell number versus time. 


\section{Results and discussion}

The production of HA using different carbon sources within $72 \mathrm{~h}$ was shown in Figure 1 - 3. The results showed that HA can be produced from glucose, sucrose and sugarcane molasses by $S$. thermophilus TISTR 458. Using of molasses as carbon sources provided the maximum HA content of $213.44 \pm 76.79 \mathrm{mg} / \mathrm{L}(12 \mathrm{~h})$ with the productivity of $569.07 \mathrm{mg} / \mathrm{g}$, while using of sucrose and glucose gave the production yield of $79.03 \pm 8.36$ and $32.80 \pm 4.27 \mathrm{mg} / \mathrm{L}$ at $24 \mathrm{~h}$, with the productivity of 84.22 and $32.80 \mathrm{mg} / \mathrm{g}$, respectively (Figure 4). An increment of HA production was related to sugar consumption which was moderately decreased.

The cell number of $S$. thermophilus TISTR 458 in biotransformation medium contained different carbon sources at each interval time were presented. Microbial cells rapidly increased, then gradually declined over time. An increase in cell number was correlated with the production yield of HA When the cell growth decreased within $24 \mathrm{~h}$ for glucose and sucrose and $12 \mathrm{~h}$ for molasses, the gradual depletion of HA production was observed.

HA was first isolated from group A hemolytic streptococci, yielding $60-140 \mathrm{mg} / \mathrm{L} \mathrm{[15].} \mathrm{Since} \mathrm{that}$ time, several studies to increase the production of HA have been consecutively developed, including the searching of effective microbial strains, optimization of culture media, conditions and the extraction process and production of high molecular weight of HA [3].

S. thermophilus YIT 2084, a generally recognized safe bacterium from dairy food products, was reported as a novel strain with high productivity of HA [8]. Afterwards, group C streptococci which gave high productivity of hyaluronan are used instead of Group A streptococci such as $S$. equi subsp. equi and S. equi subsp. Zooepidemicus [3].

Various carbon sources such as starch, lactose, glucose, sucrose and dextrin were used for HA production. In general, glucose was used as a carbon source for HA production by Streptococci strains. However, using low costs carbon sources like starch, lactose [3] and by-products from agroindustry are important goals to produce HA economically.

Previous investigations of HA production by microbial fermentation utilized various carbon and nitrogen sources from agriculture products and their by-products were reported. Production of HA by $S$. zooepidemicus ATCC 39920 using agricultural resource derivatives such as hydrolysate soy protein concentrate, whey protein concentrates and cashew apple juice was presented. The highest amount of HA of $0.89 \mathrm{~g} / \mathrm{L}$ was obtained from the cashew apple juice [6]. The productivity of HA of $0.28 \mathrm{mg} / \mathrm{g} / \mathrm{h} \mathrm{by} S$. zooepidemicus was also available from cashew apple bagasse in solid-state fermentation [15]. Moreover, Izawa et al. [8] have produced HA in the cultivation of isolated S. thermophilus YIT 2084, a generally recognized safe bacterium, in skimmed milk yielding HA productivity of approximately $8 \mathrm{mg} / \mathrm{L}$.

Molasses, agro-industrial byproducts from the sugarcane industry, consisted of various substances such as sucrose, glucose, fructose, some nitrogen sources and minerals [17,18]. Pan et al. [19] reported the production of HA of $0.376 \mathrm{~g} / \mathrm{L}$ from sugarcane molasses by $S$. zooepidemicus ATCC 39920 which was closed to that obtained from glucose $(0.429 \mathrm{~g} / \mathrm{L})$ and sucrose $(0.488 \mathrm{~g} / \mathrm{L})$. However, the maximum productivity of HA of $0.066 \mathrm{~g} / \mathrm{g}$ was from molasses.

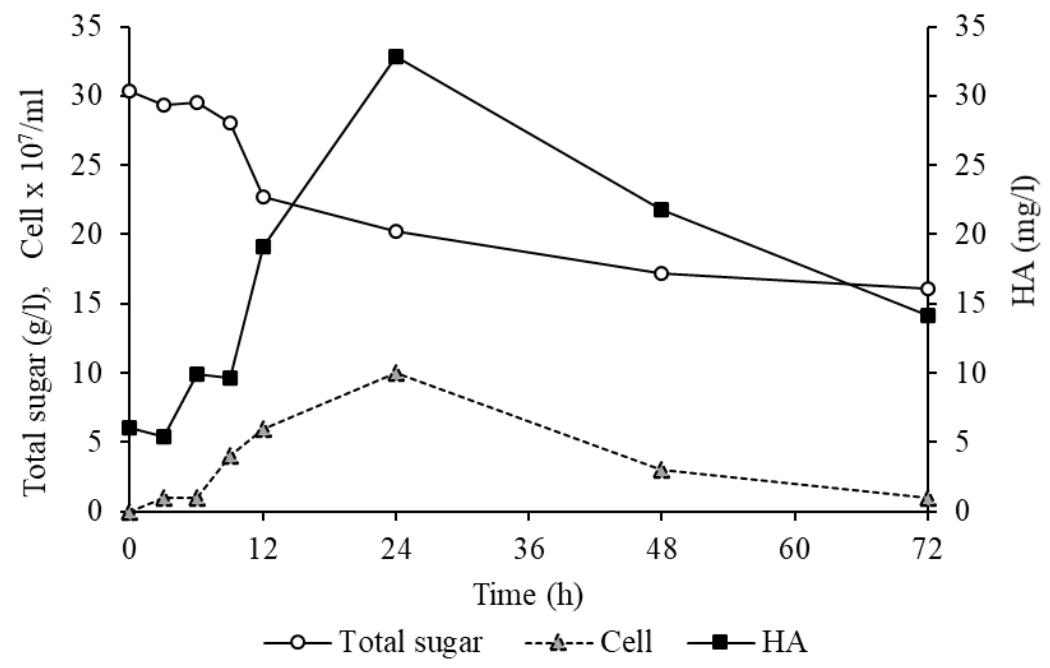

Figure 1 HA production from glucose by $S$. thermophilus TISTR 458. Results are represented as mean \pm $\operatorname{SE}(n=3)$. 


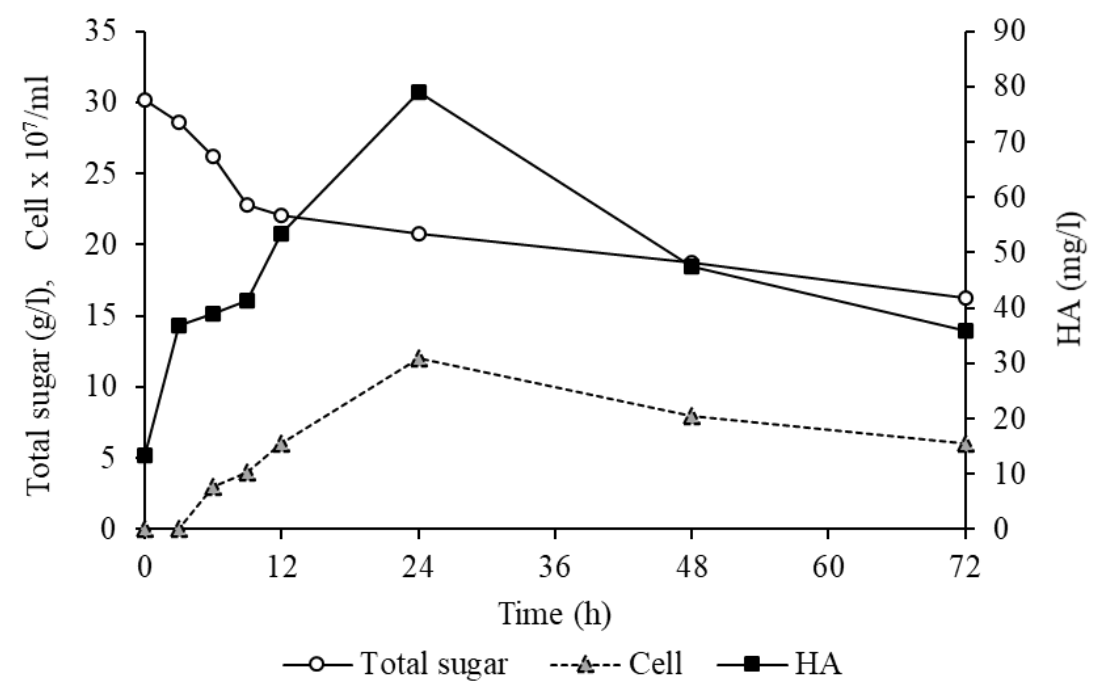

Figure 2 HA production from sucrose by $S$. thermophilus TISTR 458. Results are represented as mean \pm $\mathrm{SE}(\mathrm{n}=3)$.

From our study, we produced HA from various sugar using $S$. thermophilus TISTR 458. $S$. thermophilus belongs to the generally recognized as safe (GRAS) group which is suitable for HA production since its safety [8]. The optimal medium composed of yeast extract $30, \mathrm{~K}_{2} \mathrm{HPO}_{4} 2.5, \mathrm{NaCl} 2.0$ and $\mathrm{MgSO}_{4} \cdot 7 \mathrm{H} 2 \mathrm{O} 1.5 \mathrm{~g} / \mathrm{L}$, using sugarcane molasses as carbon source with a temperature of $37 \pm 2{ }^{\circ} \mathrm{C}$, $\mathrm{pH}$ of 6.8 . Under this condition, the production yield was $213.44 \pm 76.79 \mathrm{mg} / \mathrm{L}$ with a productivity of $5.69 \mathrm{mg} / \mathrm{g}$. This was lower than the production yield obtained by Pan et al. [19] which have also used molasses as carbon sources but higher than that from Izawa et al. [8] which have utilized S. thermophilus YIT 2084 in the fermentation. These might be due to the variation of strain effectiveness and the suitability of a carbon source for each microbial strain. Some nitrogen sources and minerals contained in molasses might influence microbial metabolism [17,18] resulted to promote hyaluronic production. However, a gradual increase of HA content during the fermentation might affect microbial growth inhibition, reflecting a further reduction of hyaluronic in culture medium while the cell growth pattern declined.

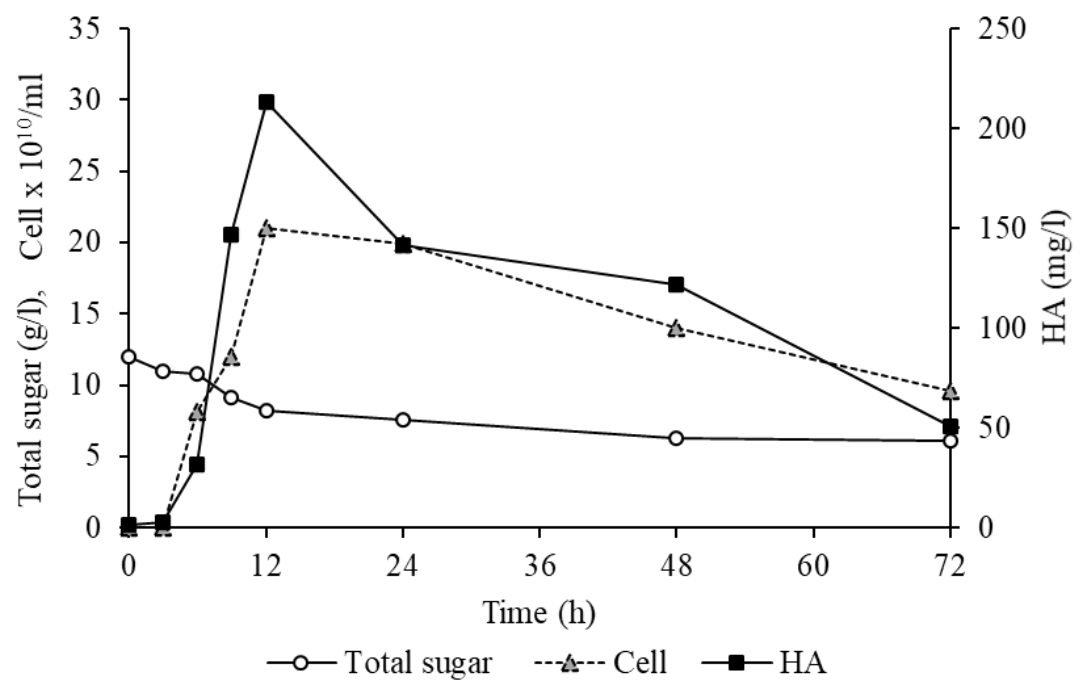

Figure 3 HA production from sugarcane molasses by S. thermophilus TISTR 458. Results are represented as mean $\pm \mathrm{SE}(\mathrm{n}=3)$. 


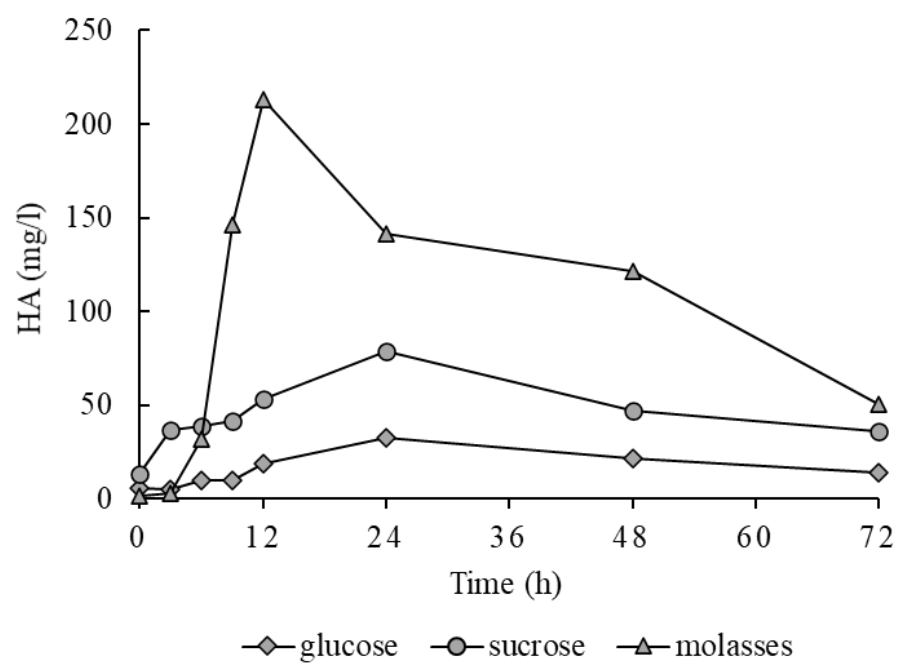

Figure 4 Comparison of HA production from glucose, sucrose and sugarcane molasses by $S$. thermophilus TISTR 458. Results are represented as mean $\pm \mathrm{SE}(\mathrm{n}=3)$.

Recently, there were interesting investigations of the production of high molecular weight HA by $S$. zooepidemicus using agro-industrial by-products including corn steep liquor and sugarcane molasses in the bioreactor. Amado et al. [20] achieved HA production of $3.48 \mathrm{~g} / \mathrm{L}$ in culture medium containing $10 \%$ $\mathrm{v} / \mathrm{v}$ corn steep liquor, as the main nitrogen source, and glucose in a batch bioreactor by $S$. zooepidemicus. The inhibition $S$. zooepidemicus growth and HA production were observed when using $10 \% \mathrm{v} / \mathrm{v}$ molasses as carbon source. Pan et al. [21] investigated the improvement of hyaluronic production by $S$. zooepidemicus ATCC 39920 in the medium containing sugarcane molasses $(85.35 \mathrm{~g} / \mathrm{L}$ total sugar) pretreated with activated charcoal and yeast extract $(50 \mathrm{~g} / \mathrm{L})$. They obtained the highest HA yield of 2.825 $\mathrm{g} / \mathrm{L}$. To optimize the polymer production using statistical techniques, Pan et al. [22] used factorial design and response surface to evaluate the concentration of various ingredients in culture medium to produce HA by $S$. zooepidemicus in shake flasks. The best medium composed of $50 \mathrm{~g} / \mathrm{L}$ of yeast extract and sucrose and $0.6 \mathrm{~g} / \mathrm{L}$ of glutamine, glutamate and oxalic acid. Under this condition, HA production in bioreactor reached the maximum yield of $0.860 \mathrm{~g} / \mathrm{L}$, with an increase of $34 \%$ productivity when compared to shake flask. These might be further applied to our study to improve HA production by $S$. thermophilus TISTR 458 by optimization of culture medium, fermentation conditions and elucidation of HA.

\section{Conclusions}

Searching for alternatives sources such as agro-industrial by-products for the production of biomolecules has been an increasing trend nowadays. Molasses was an economic source from agroindustrial by-products using as carbon sources in this study. The results expressed the promising strategy of using sugarcane molasses to produce high value-added products such as HA by $S$. thermophilus TISTR 458, a culture collection from Thailand, instead of glucose or sucrose. This fermentation product could be applied as material in food, pharmaceutics and cosmetic.

\section{Acknowledgements}

The authors gratefully acknowledge financial support from the National Research Council of Thailand through Naresuan University (grant no. R2562B004) and the lab assistance from Chalita Phophinet and Natthida Gamrai, Faculty of Pharmaceutical Sciences, Naresuan University, Thailand. 


\section{References}

[1] JH Kim, MJ Moon, DY Kim, SH Heo and YY Jeong. Hyaluronic acid-based nanomaterials for cancer therapy. Polymers (Basel) 2018; 10, 1133.

[2] KJ Reddy and KT Karunakaran. Purification and characterization of hyaluronic acid produced by Streptococcus zooepidemicus strain 3523-7. J. BioSci. Biotech. 2013; 2, 173-9.

[3] CG Boeriu, J Springer, FK Kooy, LAMVD Broek and G Eggink. Production methods for hyaluronan. Int. J. Carbohydr. Chem. 2013; 2013, 624967.

[4] G Kogan, L Soltes, R Stern and P Gemeiner. Hyaluronic acid: A natural biopolymer with a broad range of biomedical and industrial applications. Biotechnol. Lett. 2007; 29, 17-25.

[5] V Rangaswamy and D Jain. An efficient process for production and purification of hyaluronic acid from Streptococcus equi subsp. zooepidemicus. Biotechnol. Lett. 2008; 30, 493-6.

[6] AMB Pires, AC Macedo, SY Eguchi and MHA Santana. Microbial production of hyaluronic acid from agricultural resource derivatives. Bioresour. Technol. 2010; 101, 6506-9.

[7] J Mei, Z Dong, Y Yi, Y Zhang and G Ying. A simple method for the production of low molecular weight hyaluronan by in situ degradation in fermentation broth. e-Polymers $2019 ; \mathbf{1 9}$, 477-81.

[8] N Izawa, T Hanamizu, R Iizuka, T Sone, H Mizukoshi, K Kimura and K Chiba. Streptococcus thermophilus produces exopolysaccharides including hyaluronic acid. J. Biosci. Bioeng. 2009; 107, 119-23.

[9] PVM Thien and NHK Tu. Hyaluronan production in Streptococcus thermophilus. J. Chem. Pharm. Res. 2016; 8, 491-4.

[10] RG Ferreira, AR Azzoni, MHA Santana and D Petrides. Techno-economic analysis of a hyaluronic acid production process utilizing Streptococcal fermentation. Processes 2021; 9, 241.

[11] E Marcellin, W Chen and LK Nielsen. Microbial hyaluronic acid biosynthesis. In: BHA Rehm (Ed.). Microbial production of biopolymers and polymer precursors: Applications and perspectives. Caister Academic Press, Norfolk, 2009, p. 163-80.

[12] L Liu, J Sun, W Xu, M Wang, G Du and J Chen. Modelling and optimization of microbial hyaluronic acid production by Streptococcus zooepidemicus using radial basis function neural network coupling quantum-behaved particle swarm optimization algorithm. Biotechnol. Prog. 2009; 25, 1819-25.

[13] JM Song, JH Im, JH Kang and DJ Kang. A simple method for hyaluronic acid quantification in culture broth. Carbohydr. Polym. 2009; 78, 633-4.

[14] AA Albalasmeh, AA Berhe and TA Ghezzehei. A new method for rapid determination of carbohydrate and total carbon concentrations using UV spectrophotometry. Carbohydr. Polym. 2013; 97, 253-61.

[15] FE Kendall, M Heidelberger and MH Dawson. A serologically inactive polysaccharide elaborated by mucoid strains of group A hemolytic Streptococcus. J. Biol. Chem. 1937; 118, 61-9.

[16] ACD Macedo and MHA Santana. Hyaluronic acid depolymerization by ascorbate-redox effects on solid state cultivation of Streptococcus zooepidemicus in cashew apple fruit bagasse. World J. Microbiol. Biotechnol. 2012; 28, 2213-9.

[17] MRD Oliveira, RSSFD Silva, JB Buzato and MAPC Celligoi. Study of levan production by Zymomonas mobilis using regional low-cost carbohydrate sources. Biochem. Eng. J. 2007; 37, 17783.

[18] $\mathrm{K} \mathrm{Xu}$ and $\mathrm{P} \mathrm{Xu}$. Efficient production of 1-lactic acid using co-feeding strategy based on cane molasses/glucose carbon sources. Bioresour. Technol. 2014; 153, 23-9.

[19] NC Pan, JA Vignoli, C Baldo, HCB Pereira, RSDFD Silva and MAPC Celligoi. Agro-industrial byproducts for the production of hyaluronic acid by Streptococcus zooepidemicus ATCC 39920. Int. J. Sci. Technol. Res. 2015; 4, 114-8.

[20] IR Amado, JA Vazquez, L Pastrana and JA Teixeira. Microbial production of hyaluronic acid from agro-industrial by-products: Molasses and corn steep liquor. Biochem. Eng. J. 2017; 117, 181-7.

[21] N Pan, HCB Pereira, MLCD Silva, AFD Vasconcelos and MAPC Celligoi. Improvement production of hyaluronic acid by Streptococcus zooepidemicus in sugarcane molasses. Appl. Biochem. Biotechnol. 2017; 182, 276-93.

[22] NC Pan, G Biz, C Baldo and MAPC Celligoi. Factorial design in fermentation medium development for hyaluronic acid production by Streptococcus zooepidemicus. Acta Sci. Technol. 2019; 42, e42729. 